\title{
Olfactory dysfunction in Parkinson's disease: Benefits of quantitative odorant examination
}

This article was published in the following Dove Press journal:

International Journal of General Medicine

29 June 2010

Number of times this article has been viewed

Yuji Kawase'

Kazuko Hasegawa ${ }^{2}$

Noriko Kawashima ${ }^{3}$

Emiko Horiuchi ${ }^{2}$

Ken Ikeda'

'Department of Neurology, Toho University Omori Medical Center, Tokyo; ${ }^{2}$ Department of Neurology, Sagamihara National Hospital, Kanagawa; ${ }^{3}$ Kawashima Neurology Clinic, Kanagawa, Japan
Correspondence: Ken Ikeda Department of Neurology, Toho

University Omori Medical Center, 6- I I-I, Omorinishi, Otaku, Tokyo, I43-854I, Japan Tel +8I 337624 I5 I

Fax +8I 337682566

Email keni@med.toho-u.ac.jp
Abstract: Olfactory involvement is well recognized in patients with Parkinson's disease (PD). The purpose of this study was to examine smell function quantitatively, using different types and concentrations of odorants in PD patients. We aimed to elucidate whether a specific odor can affect the severity and duration of PD patients. A total of 89 nondemented PD patients and 20 age-matched controls participated in the study. Quantitative evaluation of smell function was performed using the $\mathrm{T}$ and $\mathrm{T}$ olfactometer test. This test contains five kinds of odorants at different concentrations. Recognition threshold (RT) scores for all five odorants and for each individual odorant were measured in five groups of PD patients with Hoehn and Yale (HY) stages I $(n=12)$, II $(n=24)$, III $(n=43)$, and IV $(n=10)$, as well as in control subjects $(n=20)$. One-way analysis of variance and Ryan's method were used for statistical comparison between the five groups. Compared with controls and HY I patients, total RT scores were significantly higher in HY II, III, and IV patients. There were no statistically significant differences in RT scores between HY I patients and controls. However, total RT scores for three HY I patients $(25 \%)$ were higher than the mean + two standard deviations of controls. On single odorant testing, significant higher RT scores for methylcyclopentenolone and skatol were found in HY II, III, and IV patients, in comparison with controls and HY I patients. The remaining three odorants did not differ statistically between PD patients and control subjects. The present study indicated that hyposmia in PD patients increased from HY II onwards. A single odorant of methyl cyclopentenolone or skatol had benefits for olfactory evaluation in PD patients. Our data also clarified that olfactory deficits occurred in a subset of HY I patients. Further prospective study is needed to elucidate whether a distinct profile of PD exists between HY I patients with and without hyposmia.

Keywords: Parkinson's disease, Hoehn and Yale stage, olfactory dysfunction, odorants

\section{Introduction}

Olfactory impairment in Parkinson's disease (PD) was first described by Ansari and Johnson. ${ }^{1}$ The frequency of olfactory dysfunction is $70 \%-90 \%$ in PD patients, ${ }^{2}$ and rates of hyposmia correspond to presence of tremor. ${ }^{3}$ Neuropathologic research in PD patients suggests neuronal damage in the olfactory system, including the olfactory bulb and the anterior olfactory nucleus. ${ }^{4}$ There have been many studies of the relationship between olfactory deficits and PD progression..$^{5-8}$ Several studies showed that PD progression was correlated with olfactory dysfunction. ${ }^{6-8}$ Tissingh et $\mathrm{al}^{7}$ pointed out that odor discrimination was negatively associated with the Unified Parkinson's Disease Rating Scale (UPDRS) and Hoehn and Yale (HY) stage. This suggests that olfactory tests may have benefits for early diagnosis of PD. Previous olfactory tests used single or 
several kinds of odorants. ${ }^{5-8}$ The T and T olfactometer (TTO) test is a quantitative smell test that contains five odorants and different concentrations of each odorant. The present study was designed to evaluate how useful TTO is as a tool for detection of hyposmia in PD patients and the relationship between hyposmia and disease progression.

\section{Methods}

\section{PD patients and control subjects}

PD was diagnosed according to the UK Parkinson's Disease Society Brain Bank criteria. A total of 89 patients with PD and 20 age-matched healthy controls participated in the study (see Table 1). Mean age ( \pm standard deviation, SD) was 69.1 (7.1) years in PD patients and 68.3 (8.2) years in controls. Mean disease duration was 5.6 (4.6) years. PD severity was classified by HY criteria, and mean HY stage was 2.5 (0.9). Patients were categorized as HY I $(n=12)$, II $(n=24)$, III $(n=43)$ and IV $(n=10)$. PD patients with dementia, defined according to Mini Mental State Examination scores lower than 22 points, were excluded. Before the smell examination, all PD patients had had an otorhinolaryngology consultation, and those with suspected rhinologic disorders underwent further examination, including nasal endoscopy. PD patients and control subjects who had severe rhinologic disease causing respiratory hyposmia, such as chronic or acute sinusitis, allergic rhinitis, nasal polyps, tumors, or severe septal deviation were excluded. PD patients with olfactory hallucinations on rhinologic examination were also excluded. The present study was performed and approved according to the clinical guidelines of Sagamihara National Hospital. Informed consent was obtained from all study participants.

\section{Quantitative olfactory test}

Olfactory function was evaluated using the TTO test (Takasago Co., Tokyo). Olfactory examination was undertaken in a special odorless room. The temperature of the room was adjusted to $23^{\circ} \mathrm{C}$. Adequate air ventilation was maintained. Previous data show that the results of this test have a good correlation with the University of Pennsylvania Smell Identification Test (UPSIT). ${ }^{9}$ The TTO test used five kinds of odorants, comprising A ( $\beta$-phenyl ethyl alcohol), B (methyl cyclopentenolone), C (isovaleric acid), $\mathrm{D}$ ( $\gamma$-undecalactone), and $\mathrm{E}$ (skatol). The A odor was rosescented ("rose"), B charred ("caramel"), C rancid ("putrefaction"), D peach-like ("peach"), and E halitosis-like ("feces"). Seven or eight sequential dilutions of each odorant were prepared. Each odorant was judged by a numeric score of $-2-5$ according to its concentration. Each odor test was performed gradually from the lowest concentration $(-2)$ to higher concentrations. The subject was asked to sniff a paper edge soaked in an odorant and to describe the nature of the odor. Recognition threshold (RT) was defined as the lowest concentration at which a subject described the type of odor correctly. A patient could answer "rose" or "sweet-scented flower" for the odorant A test. Similarly, the correct response to odorant B was "caramel" or "burnt sugar". Odorant C could be answered as "rancid", "rotten food" or "sweaty clothes". Odorant D could be answered as "peach-like" or "sweet fruit". Odorant E could be answered as "halitosis-like", "feces" or "kitchen". The test was repeated from odorant $\mathrm{A}$ to $\mathrm{E}$ in sequence. When a patient failed to identify the odor at the highest possible concentration of odors A to E accurately, the score was determined as six points. Total RT was calculated as the average of five odorant scores $([\mathrm{A}+\mathrm{B}+\mathrm{C}+\mathrm{D}+\mathrm{E}] / 5)$. A larger $\mathrm{RT}$ value indicated more severe olfactory dysfunction. The detection threshold (DT) of the TTO test was assessed only by the presence or absence of smell. Therefore, DT did not canvas the correct type or character of each odorant. DT was therefore not used for quantitative evaluation of olfactory function in this study.

\section{Statistical analysis}

All data were shown as mean (SD). One-way analysis of variance (ANOVA) and Ryan's method were used to compare RT scores for the four PD groups of HY I-IV patients and the control group. After one-way ANOVA, Ryan's method was performed for multiple comparisons. The level of significance was set at $P<0.05$ for both tests.

Table I Clinical profile of Parkinson's disease patients and control subjects

\begin{tabular}{|c|c|c|c|c|c|c|}
\hline & Control $(n=20)$ & Total & HY I (n = I2) & HY II $(n=24)$ & HY III $(n=43)$ & HY IV $(n=10)$ \\
\hline Male/female & $5 / 15$ & $35 / 54$ & $5 / 7$ & $11 / 13$ & $16 / 27$ & $3 / 7$ \\
\hline Mean age (SD) years & $68.3(8.2)$ & $69.1(7.1)$ & $68.2(6.8)$ & $67.0(7.8)$ & $70.5(7.9)$ & $69.5(9.5)$ \\
\hline Mean duration of PD (SD) years & & $5.6(4.6)$ & $1.9(1.6)$ & $4.8(3.6)$ & $5.8(4.4)$ & II.0 (5.6) \\
\hline Mean MMSE score (SD) & $27.6(2.2)$ & $25.8(2.8)$ & $26.8(2.9)$ & $26.0(3.1)$ & $25.1(2.3)$ & $25.8(2.9)$ \\
\hline
\end{tabular}

Abbreviations: HY, Hoehn and Yale; MMSE, mini-mental state examination; PD, Parkinson's disease; SD, standard deviation. 


\section{Total RT score}

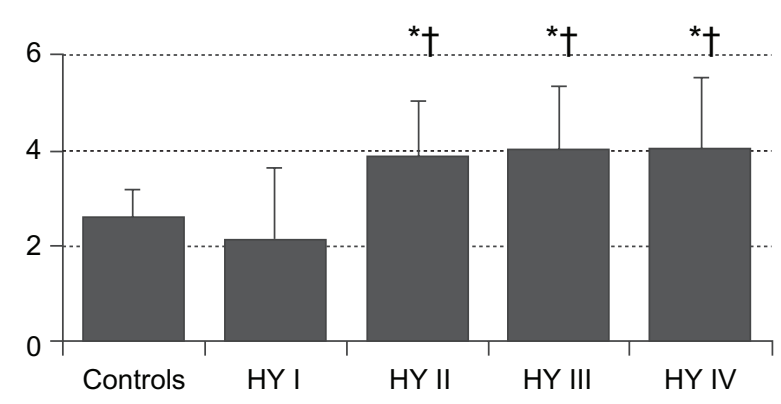

Figure I Total RT scores of five odorants in PD patients and control subjects. Compared with controls or HY I patients, total RT scores were higher in HY II, III, and IV patients ( $F=8.154$ and $P<0.001$ by one-way ANOVA).

Notes: $* P<0.0$ I by Ryan's method among the PD subgroups of HY II, III, and IV, and the control group; ${ }^{\dagger} P<0.0$ l by Ryan's method between the HY I group and other HY groups.

Abbreviations: ANOVA, one-way analysis of variance; HY, Hoehn and Yale; PD, Parkinson's disease; RT, recognition threshold.

\section{Results}

The mean value (SD) of total RT scores were $2.6(0.6)$ in control subjects, 2.1 (1.5) in HY I, 3.8 (1.2) in HY II, 3.9 (1.4) in HY III, and 3.9 (1.6) in HY IV patients (Figure 1). Total RT scores were significantly higher in the HY II, HY III, and HY IV groups compared with the control group and the HY I group $(\mathrm{F}=8.154, P<0.001$ by one-way ANOVA, $P<0.01$ by Ryan's method). These results suggest that olfactory dysfunction occurred in $\mathrm{PD}$ patients with HY II disease onwards. Three HY I patients $(25 \%)$ had higher than the mean +2 SD total RT scores for controls. Mean RT scores of odorant A were 3.4 (1.4) in controls, 3.3 (2.7) in HY I, 4.8 (1.8) in HY II, 4.8 (1.8) in HY III, and 4.0 (2.3) in HY IV patients. These scores did not differ between the four groups of HY I-IV patients and controls (Figure 2A). Mean RT scores of odorant B were $2.2(1.3)$ in controls, 1.8 (1.8) in HY I, 3.9 (1.7) in HY II, 3.9 (1.6) in HY III, and 4.6 (1.5) in HY IV patients

\section{Odor A: $\beta$-phenyl ethyl alcohol}

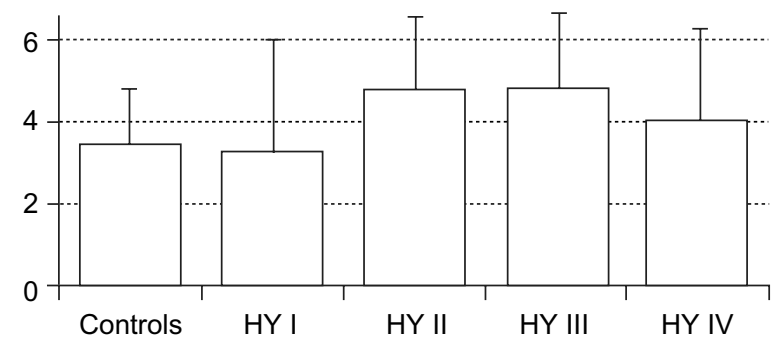

Figure 2A RT score of odorant A. There were no significant differences of odorant A scores between the five groups.
Odor B: methyl cyclopentenolone

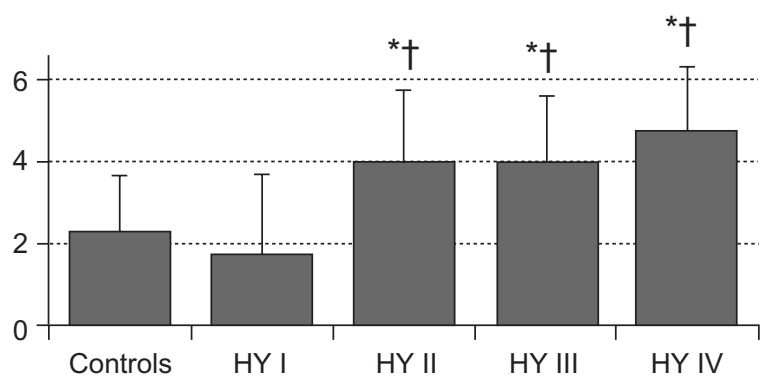

Figure 2B RT score of odorant B. Compared with controls and HY I patients, RT scores for odorant $B$ were significantly higher in $\mathrm{HY} I \mathrm{I}$, III, and IV patients. $(F=8.848$, $P<0.00$ I by one-way ANOVA).

Notes: $* P<0.01$ by Ryan's method between the PD HY II, III, and IV subgroups and controls; ${ }^{\dagger} P<0.0$ I by Ryan's method between the HY I group and other HY groups.

(Figure 2B). RT scores of HY II, III, and IV patients were significantly higher than those of controls and HY I patients ( $\mathrm{F}=8.848, P<0.001$ by one-way ANOVA, $P<0.01$ by Ryan's method). Mean RT scores of odorant $C$ were 2.0 (1.2) in controls, 1.0 (1.6) in HY I, 3.3 (1.8) in HY II, 3.2 (1.7) in HY III, and 3.0 (1.9) in HY IV patients (Figure 2C). Compared with HY I patients, RT scores of odorant $\mathrm{C}$ were increased significantly in HY II, III, and IV patients ( $\mathrm{F}=16.294, P<0.001$ by one-way ANOVA, $P<0.01$ by Ryan's method). These scores did not differ statistically between any of the four PD subgroups and controls. Mean scores of odorant D were 3.0 (1.3) in controls, 2.6 (2.1) in HY I, 3.0 (1.7) in HY II, 3.7 (1.9) in HY III, and 3.8 (2.2) in HY IV patients (Figure 2D). RT scores of odorant D did not differ significantly between the HY I, II, III, and IV patients and controls. Mean scores of odorant E were 1.8 (1.0) in controls, 2.1 (2.0) in HY I, 4.1 (2.1) in HY II, 4.0 (2.1) in HY III, and 4.3 (2.0) in HY IV patients (Figure 2E). Those scores were significantly higher in HY II, III, and IV patients than in controls or HY I patients ( $\mathrm{F}=27.876, P<0.001$ by one-way ANOVA, $P<0.01$ by Ryan's method).

\section{Odor C: isovaleric acid}

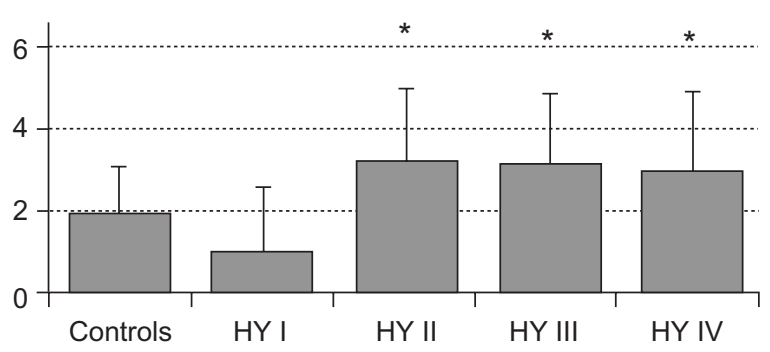

Figure 2C RT score of odorant C. Compared with HY I patients, RT scores of odorant C were increased significantly in HY II, III, and IV patients ( $F=16.294$ and $P<0.00$ I by one-way ANOVA).

Note: $* P<0.0$ I by Ryan's method. 


\section{Odor D: $\gamma$-undecalactone}

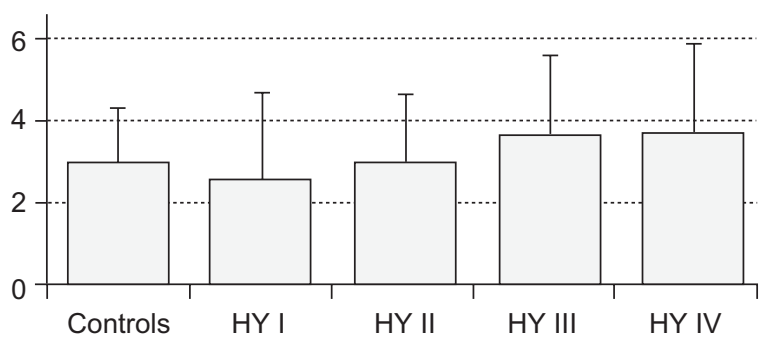

Figure 2D RT score of odorant D. There were no significant differences of odorant $\mathrm{D}$ scores between the five groups.

\section{Discussion}

The present study showed that olfactory function in PD patients declined in a statistically significant manner from $\mathrm{HY}$ II onwards. There were no statistically significant differences between HY I patients and controls, although three of 12 patients with HY I had hyposmia in comparison with controls. Comparative analyses of five odorants revealed that odorant $\mathrm{B}$ (methyl cyclopentenolone) and E (skatol) were involved in PD patients with HY II-IV. RT scores of odorant C (isovaleric acid) were significantly lower in HY I than in HY II, III, and IV patients, whereas no statistically significant differences existed between the HY stage PD subgroups and controls.

The association between olfactory deficits and PD progression has been controversial in previous reports. On UPSIT, a classical olfactory test, olfactory dysfunction was not correlated with PD severity. ${ }^{5}$ A recent longitudinal study found no relationship between olfactory function and PD progression, including patient age, PD stage, and duration. Some PD patients have been found to recover from olfactory deficits on Sniffin Stick testing. ${ }^{10}$ Other studies have found a significant association between hyposmia and PD stage..$^{6,7}$ Olfactory function was shown to be impaired in patients with more than HY III than in those with HY I and II disease. ${ }^{6}$ Odor discrimination was lost with motor disease progression according to UPDRS and HY classification. ${ }^{7}$ Serial olfactory examination using the Sniffin Stick test was performed in five de novo PD patients. Olfactory function worsened in three patients, and the degree of hyposmia was attenuated in one patient. ${ }^{8}$ The present study revealed statistically significant differences in olfactory function between HY II, III, and IV PD patients and controls. Three HY I patients (25\%) also showed hyposmia. Predictive studies of hyposmia in PD have also been reported. ${ }^{11-14}$ Of 361 asymptomatic Dutch relatives of PD patients, 40 had hyposmia. After two years of follow-up, four hyposmic relatives $(10 \%)$ developed PD. ${ }^{12}$ In another Germany study of 30 patients with idiopathic

\section{Odor E: skatol}

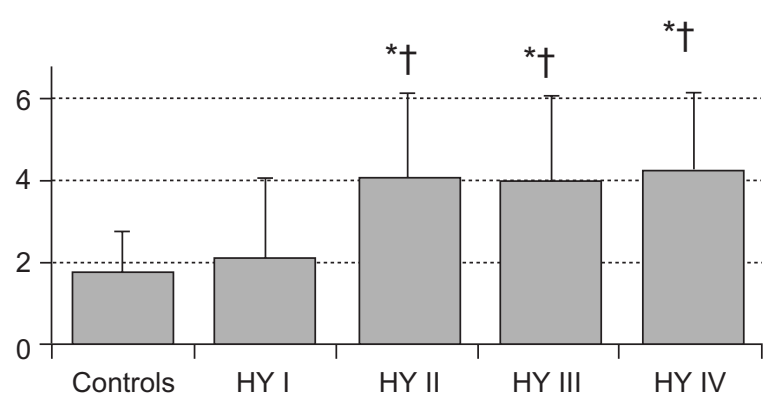

Figure 2E RT score of odorant E. Compared with controls and HY I patients, RT scores for odorant $E$ were higher significantly in HY II, III, and IV patients ( $F=27.876$ and $P<0.001$ by one-way ANOVA).

Notes: $* P<0.0$ I by Ryan's method between the PD HY II, III and IV subgroups and controls; ${ }^{\dagger} P<0.0$ I by Ryan's method between the HY I group and other HY groups.

Abbreviations: ANOVA, one-way analysis of variance; HY, Hoehn and Yale; $\mathrm{PD}$, Parkinson's disease; RT, recognition threshold.

hyposmia, PD developed in two patients (7\%) during two years of follow-up. ${ }^{13}$

In addition to those patients with preclinical PD and signs of hyposmia, the present study supports the possibility that smell dysfunction could start in a subset of HY I patients. Previous neuropathologic research has mentioned that dopaminergic neurons in the olfactory bulb are potentiated in the early stages of PD by a compensatory mechanism against the loss of dopaminergic neurons in the basal ganglia. ${ }^{15}$ Such dopaminergic changes in the olfactory bulb may contribute to deteriorating olfactory function in PD patients. Thereafter, dopaminergic neuronal loss occurs in the olfactory bulb, so hyposmia may improve in patients with advanced PD. This hypothesis does not suggest a constant relationship between olfactory function and motor dysfunction in PD patients.

The UPSIT and Sniffin Stick tests use only one concentration for each odorant. In these tests, a single odorant is judged by only one response as presence ("yes") or absence ("no"). Odorants of wintergreen and pizza are distinguishable on UPSIT, and those odors show more sensitivity and specificity in PD patients. ${ }^{16}$ The Brief Smell Identification Test (BSIT), a simplified version of UPSIT, uses five kinds of odorants (gasoline, banana, pineapple, smoke, and cinnamon). This smell test has shown that several odorants could be used to discriminate between PD patients and normal subjects. ${ }^{17}$ Previous reports of use of these studies have provided different sensitivities for different types of odorants in PD patients. The advantage of these tests is that they are easy and convenient to administer, but the olfactory data include no quantitative evaluation using different concentrations of odorant. ${ }^{16,17}$ 
In comparison with the conventional smell tests used in Europe and the US, the TTO test was devised as a quantitative odor assessment method in Japan. Methodologic features of the TTO test include five odorants and seven or eight different concentrations of each odorant. A pilot TTO study showed that total RT score differed significantly between PD patients and age-matched controls, although PD stage and RT score were not analyzed for each odorant. ${ }^{18}$ In another small study of the TTO test in 10 PD patients and 10 controls, only two odorants were investigated, one pleasant and one unpleasant. ${ }^{19}$ In our study, we have analyzed olfactory function in a large number of PD patients $(n=89)$ by TTO examination using five odorants. Two kinds of odorants were found to be useful for olfactory evaluation in these patients. Although no significant correlation existed between smell and motor dysfunction, some HY I patients had hyposmia. Besides the peripheral olfactory nerves and bulb, involvement of the corticobasal pathway or the olfactory limbic system could be a possible causative mechanism for hyposmia. A recent pathologic study mentioned no significant histochemical abnormalities of the olfactory epithelium between PD patients and control subjects. ${ }^{20}$ Therefore, the most crucial pathologic damage occurring in the peripheral and central olfactory systems remains unclear in PD patients with hyposmia.

The present study using the TTO test indicates that hyposmia occurs in PD patients with HY II-IV disease. Total RT score and RT scores for methyl cyclopentenolone and skatol were useful for quantitative olfactory examination. These scores were not correlated with disease duration and HY stage in our patients. It is of interest that olfactory deficits were found in a minority of PD patients with HY I disease. Further longitudinal study is needed to clarify whether distinct clinical courses exist between HY I patients with and without hyposmia.

\section{Acknowledgments}

The authors are grateful to Professor Yasuo Iwasaki, Department of Neurology, Toho University Omori Medical Center, Tokyo, Japan for critical suggestions and support of this study.

\section{Disclosure}

The authors report no conflicts of interest in this work, financial or otherwise.

\section{References}

1. Ansari KA, Johnson A. Olfactory function in patients with Parkinson's disease. J Chronic Dis. 1975;28:493-497.

2. Daum RF, Sekinger B, Kobal G, et al. Riechpruefung mit "sniffin' sticks" zur klinischen Diagnostik des Morbus Parkinson. Nervnarzt. 2000;71:643-650.

3. Hawkes $\mathrm{CH}$, Shephard BC, Daniel SE. Is Parkinson's disease a primary olfactory disorder? QJM. 1999;56:33-39.

4. Braak H, Del Tredici K, Rüb U, et al. Staging of brain pathology related to sporadic Parkinson's disease. Neurobiol Aging. 2003;24:197-211.

5. Doty RL, Deems DA, Stellar S. Olfactory function in Parkinsonism: A general deficit unrelated to neurologic signs, disease stage, or disease duration. Neurology. 1988;38:1237-1244.

6. Stern MB, Doty RL, Dotti M, et al. Olfactory function in Parkinson's disease subtypes. Neurology. 1994;44:266-268.

7. Tissingh G, Berendse HW, Bergmans P, et al. Loss of olfaction in de novo and treated Parkinson's disease: Possible implications for early diagnosis. Mov Disord. 2001;16:41-46.

8. Müller A, Reichmann H, Livermore A, et al. Olfactory function in idiopathic Parkinson's disease (IPD): Results from cross-sectional studies in IPD patients and long-term follow-up of de novo IPD patients. J Neural Transm. 2002;109:805-811.

9. Kondo H, Matsuda T, Hashida M, et al. A study of the relationship between the T \& T Olfactometer and the University of Pennsylvania Smell Identification Test in a Japanese population. Am J Rhinol. 1998;12:353-358.

10. Herting B, Schulze S, Reichmann H, et al. A longitudinal study of olfactory function in patients with idiopathic Parkinson's disease. J Neurol. 2008;255:367-370.

11. Berendse HW, Booij J, Francot CM, et al. Subclinical dopaminergic dysfunction in asymptomatic Parkinson's disease patients' relatives with a decreased sense of smell. Ann Neurol. 2001;50:34-41.

12. Ponsen MM, Stoffers D, Booij J, et al. Idiopathic hyposmia as a preclinical sign of Parkinson's disease. Ann Neurol. 2004;61: $173-181$.

13. Haehner A, Hummel T, Hummel C, et al. Olfactory loss may be a first sign of idiopathic Parkinson's disease. Mov Disord. 2007;22:839-842.

14. Ross GW, Petrovitch H, Abbott RD, et al. Association of olfactory dysfunction with risk for future Parkinson's disease. Ann Neurol. 2008;63:167-173.

15. Huismann E, Uylings HB, Hoogland PV. A 100\% increase of dopaminergic cells in the olfactory bulb may explain hyposmia in Parkinson's disease. Mov Disord. 2004;19:687-692.

16. Hawkes CH, Shephard BC, Daniel SE. Olfactory dysfunction in Parkinson's disease. J Neurol Neurosurg Psychiatry. 1997;62: 436-446.

17. Double KL, Rowe DB, Hayes M, et al. Identifying the pattern of olfactory deficits in Parkinson disease using the brief smell identification test. Arch Neurol. 2003;60:545-549.

18. Murofushi T, Mizuno M, Osanai R, et al. Olfactory dysfunction in Parkinson's disease. ORL J Otorhinolaryngol Relat Spec. 1991;53:143-146.

19. Masaoka Y, Satoh H, Kawamura M, et al. Respiratory responses to olfactory stimuli in Parkinson's disease. Respir Physiol Neurobiol. 2008;161:136-141.

20. Witt M, Bormann K, Gudziol V, et al. Biopsies of olfactory epithelium in patients with Parkinson's disease. Mov Disord. 2009;24:906-914.
International Journal of General Medicine

\section{Publish your work in this journal}

The International Journal of General Medicine is an international, peer-reviewed open-access journal that focuses on general and internal medicine, pathogenesis, epidemiology, diagnosis, monitoring and treatment protocols. The journal is characterized by the rapid reporting of reviews, original research and clinical studies across all disease areas.

\section{Dovepress}

A key focus is the elucidation of disease processes and management protocols resulting in improved outcomes for the patient. The manuscript management system is completely online and includes a very quick and fair peer-review system. Visit http://www.dovepress.com/ testimonials.php to read real quotes from published authors. 